\title{
Competing causes of death in patients with oropharyngeal cancer treated with radiotherapy
}

\author{
JOHNNY KAO ${ }^{1,3}$, K.H. VINCENT LAU ${ }^{1}$, CHARLES C.L. TONG ${ }^{2}$ and CHIEN-TING CHEN ${ }^{1}$ \\ Departments of ${ }^{1}$ Radiation Oncology, and ${ }^{2}$ Otolaryngology-Head and Neck Surgery, Mount Sinai School of Medicine, \\ New York, NY; ${ }^{3}$ Good Samaritan Hospital Medical Center, West Islip, NY, USA
}

Received October 25, 2011; Accepted January 19, 2012

DOI: $10.3892 /$ etm.2012.494

\begin{abstract}
Radiation with or without chemotherapy is considered the mainstay of treatment for the majority of patients with oropharyngeal cancer. The goal of this study was to analyze competing causes of mortality in patients with oropharyngeal cancer with long-term follow-up. We queried the Surveillance, Epidemiology and End Results (SEER) database and identified 3728 patients with oropharyngeal cancer treated between 1988 and 2001 with definitive radiotherapy. We analyzed predictors of overall survival and risks of mortality from index oropharyngeal cancer, second primary cancer, cardiovascular disease and other causes using a cumulative incidence analysis and Cox multivariate analysis. With a median follow-up of 6.8 years, the 5- and 10-year overall survival was 37 and $22 \%$, respectively. At 5 years, the risk of mortality from primary oropharyngeal cancer was $35 \%$. Between years 3 and 10 , $69 \%$ of mortalities were attributed to causes other than the index cancer. Despite advances in the non-surgical treatment of oropharyngeal cancer, patients remain at significant risk of cancer- and non-cancer-related mortality.
\end{abstract}

\section{Introduction}

The majority of patients treated with definitive radiotherapy with or without chemotherapy in recent locally advanced head and neck cancer trials are oropharyngeal cancer patients (1-3). The incidence of base of tongue and tonsil cancer in younger patients has increased dramatically over the past three decades (4). Several strategies designed to improve locoregional control for locally advanced oropharyngeal cancers, including concurrent chemoradiotherapy, intensity modulated radiation therapy and altered fractionation radiation therapy, have been extensively investigated (5-9). However, curing the index cancer eliminates only one of several mortality risks

Correspondence to: Dr Johnny Kao, Department of Radiation Oncology, Good Samaritan Hospital Medical Center, 1000 Montauk Highway, West Islip, NY 11795, USA

E-mail: johnnykaomd@gmail.com

Key words: oropharyngeal cancer, radiation, second malignancies, competing mortality faced by these patients (10). Due to competing risks of second primary cancer and comorbid medical conditions related to smoking and alcohol use, overall survival remains consistently lower than disease-free survival (11). Recently, high-risk human papilloma virus (HPV) infection has emerged as an important risk factor for oropharyngeal carcinoma (12). HPV infection is now found in more than $50 \%$ of patients with oropharyngeal cancer and tends to affect younger patients irrespective of tobacco or alcohol use (13). Preliminary data suggest that these patients are at lower risk for second primary cancers due to the focal nature of HPV infection (14). Furthermore, since these patients tend to be younger and are less likely to be heavy users of tobacco, they may also be at lower risk for cardiovascular disease $(13,15)$. To evaluate the different causes of mortality in patients with oropharyngeal carcinoma treated with radiotherapy, we conducted an analysis of the SEER database.

\section{Materials and methods}

Patient selection. The patient population in this study consisted of adult patients with stage I-IVb oropharyngeal squamous cell carcinoma diagnosed between 1988 and 2001 in the Surveillance, Epidemiology, and End Results (SEER) 17 database, who had been treated with definitive radiotherapy. The SEER17 database is a longitudinal database that collects information from 17 cancer registries covering $26 \%$ of the US population. The SEER17 database is composed of 17 population-based cancer registries from Connecticut, New Jersey, Atlanta, Kentucky, Louisiana, rural Georgia, Detroit, Iowa, Hawaii, New Mexico, Seattle-Puget Sound, Utah, San Francisco-Oakland, San Jose-Monterey, Los Angeles, greater California and the Alaska Native Tumor Registry. Serial registry data are de-identified and submitted to the U.S. National Cancer Institute on a bi-annual basis and these data are publicly available for investigators (16). Therefore, approval by an ethics committee was not necessary to perform the analyses. The population covered by the SEER database is considered representative of the US population and the case ascertainment rate is reportedly $97.5 \%$ (16).

We identified 3728 eligible patients aged 18 to 85 with squamous cell carcinoma (SCC) of the oropharynx for this analysis. Patients were excluded if there was no histological confirmation, missing radiation records or unknown nodal 
stage. Additionally, 58\% of patients had available size and tumor extension data allowing for further classification into AJCC 6th edition T stage. Patients were all subclassified into base of tongue (C01 or C02.4), tonsil (C09), soft palate (C05.1 or C05.2), oropharyngeal wall (C10.2 or C10.3) and oropharynx NOS (C10.0, C10.1, C10.8 or C10.9) using ICD-O-2 codes. Cause of mortality was determined by SEER site recode codes, since ICD-O-2 codes were not available. Patients with mortality from oral cavity (20020, 20040 and 20050) and oropharyngeal cancer (20020, 20050, 20070 and 20080) were classified as mortality from index oropharyngeal cancer. Additionally, mortality from miscellaneous malignant cancers (37000) was classified as mortality from index cancer since $88 \%$ of these deaths occurred within 5 years of diagnosis. Patients with mortality from other types of cancer were classified as second cancers. Patients with mortality from cardiovascular disease (50060, 50070, 50080, 50090, 50100 and 50110) and other causes were scored separately. The most recent follow-up available was December 2006.

Statistical analysis. Categorical variables included age $(\leq 50$, 51-60, 61-70 and >70), date of diagnosis (1988-1994 vs. 1995 to 2001), gender, ethnicity (Caucasian/Asian vs. African descent), primary site (tonsil vs. base of tongue vs. other), T stage, lymph node stage (N1, N2a, N2b, N2c, N3), lymph node surgery, tumor size $(2 \mathrm{~cm}, 2.1-4 \mathrm{~cm}$, and $>4 \mathrm{~cm})$, tumor grade, marital status and prior cancer diagnosis (Table I). Patient age in years was analyzed as a categorical variable on univariate analysis but as a continuous variable on multivariate analysis. Information regarding HPV status, use of adjuvant chemotherapy, performance status and radiotherapy details (dose, fractionation and 3-dimensional conformal/intensity modulated radiotherapy) were not available within the SEER database and this information was not included for analysis. Overall survival was the primary endpoint and mortality from primary oropharyngeal cancer, second cancers, cardiovascular and other causes were secondary endpoints.

All analyses were performed using Stata software (version 9.1; StataCorp, College Station, TX, USA) by importing data from the SEER (available at URL: www.seer.cancer.gov; accessed on September 14, 2009) 1973-2006 Public Use Data (National Cancer Institute, April 2009 release based on the November 2008 submission) into Stata. Overall survival was calculated from the time of diagnosis to the time of mortality or last follow-up using the Kaplan-Meier method. Causespecific mortality was calculated from the time of diagnosis to the time of event or last follow-up. We analyzed the actuarial rates of cause-specific mortality using the cumulative incidence method described by Coviello et al using Stata 9.1 (17). When there are competing risks, the Kaplan-Meier method for estimation of cumulative incidence curves is considered inaccurate (18). For overall survival, the stratified log-rank test was utilized to compute survival estimates that were within specified strata levels. Results were considered to indicate a statistically significant difference at P-values $<0.05$.

Cox proportional hazards regression modeling was limited to covariates that were found to be statistically significant on univariate analysis. A multivariate Cox analysis was developed to calculate the adjusted hazards ratios (HRs) and $95 \%$ confidence intervals (95\% CIs) for 1737 patients with complete datasets. Separate multivariate models were developed for specific causes of cancer mortality. A formal examination of the proportional hazards assumption was performed graphically by plotting $-\log (\log (\mathrm{S}(\mathrm{t}))$ versus $\log (\mathrm{t})$ for each covariate. This confirmed that the covariates are independent with respect to time and their HRs are constant over the clinically relevant period of follow-up.

\section{Results}

Outcomes. The 5- and 10-year overall survival was 37 and 22\%, respectively (Fig. 1A). Median follow-up for surviving patients was 6.8 years (range, 0.1-18.8). Predictors of decreased survival on univariate analysis included advanced age, African descent, earlier date of diagnosis, non-married, prior cancer, well or moderately differentiated disease, subsite other than tonsil or base of tongue, advanced $\mathrm{T}$ stage and advanced nodal disease (Table I). Gender was not significant on univariate analysis. There was no significant difference by nodal status of N0 to $\mathrm{N} 2 \mathrm{a}(\mathrm{p}=0.14)$ or between tonsil vs. base of tongue $(\mathrm{p}=0.28)$.

Causes of mortality. To date, the causes of mortality included none $(n=942,25 \%)$, primary oropharyngeal cancer $(n=1428$, $38 \%)$, second primary cancer $(n=613,16 \%)$, cardiovascular $(n=318,9 \%)$ and other causes $(n=427,11 \%)$. Various types of mortality occurred in different patterns. The 5- and 10-year risk of mortality from primary cancer was 35 and $37 \%$ (Fig. 1B). The 5 and 10-year risk of mortality from second primary cancers was 16 and $21 \%$, respectively (Fig. 1C). The 5- and 10-year risk of cardiovascular mortality was 5 and $9 \%$ (Fig. 1D). The 5- and 10-year risk of mortality from other causes was 7 and $11 \%$ (Fig. 1E). While $60 \%$ of mortalities within the first 3 years of diagnosis of cancer were classified as oral cavity or oropharyngeal cancers, $69 \%$ of mortalities after 3 years were related to second primaries, cardiovascular disease or second primary cancers.

Multivariable analyses. On Cox regression analysis, all available prognostic factors were predictive of overall survival (Table II). The strongest predictors of decreased survival in order of significance, were advanced age, African descent, single marital status, advanced $\mathrm{T}$ stage, advanced $\mathrm{N}$ stage and well/moderately differentiated disease. Separate multivariable analyses of causes of mortality demonstrate that different prognostic factors are implicated (Table III). Age, advanced nodal disease, advanced $\mathrm{T}$ stage, well/moderately differentiated disease, African descent and unmarried status were associated with greater risk of mortality from primary oropharyngeal cancer. Predictors of mortality from second cancer were nonHPV-associated subsite, prior cancer diagnosis, advanced age, male gender and well/moderately differentiated disease. Risk factors associated with mortality from cardiovascular disease were advanced age, African ethnicity, male gender and prior diagnosis of cancer. Only advanced age and unmarried status predicted for mortality from other causes.

\section{Discussion}

Treatment of primary oropharyngeal cancer. Despite advances in non-surgical therapy for oropharyngeal cancer 
Table I. Patient and tumor characteristics.

\begin{tabular}{|c|c|c|c|c|}
\hline $\begin{array}{l}\text { Variable } \\
\text { Age (years) }\end{array}$ & $\mathrm{n}(\%)$ & \multicolumn{2}{|c|}{ 5-year overall survival (\%)10-year overall survival (\%) } & \multirow{2}{*}{$\begin{array}{r}\text { P-value } \\
<0.001\end{array}$} \\
\hline Age (years) & median 63 (range 18-85) & & & \\
\hline$\leq 50$ & $577(15)$ & 49 & 37 & \\
\hline $51-60$ & $1033(28)$ & 43 & 31 & \\
\hline $61-70$ & $1136(30)$ & 36 & 21 & \\
\hline$>70$ & $982(26)$ & 26 & 9 & \\
\hline Ethnicity & & & & $<0.001$ \\
\hline Caucasian & $2797(75)$ & 40 & 24 & \\
\hline African descent & $546(15)$ & 24 & 13 & \\
\hline Asian & 161 (4) & 45 & 36 & \\
\hline Hispanic & 207 (6) & 39 & 24 & \\
\hline Other & $18(0)$ & 41 & 33 & \\
\hline Gender & & & & 0.18 \\
\hline Male & $2726(73)$ & 37 & 22 & \\
\hline Female & $1002(27)$ & 39 & 24 & \\
\hline Date of diagnosis & & & & $<0.001$ \\
\hline $1988-1994$ & $1297(35)$ & 32 & 17 & \\
\hline $1995-2001$ & $2431(65)$ & 41 & 27 & \\
\hline Marital status & & & & $<0.001$ \\
\hline Married & $1807(48)$ & 44 & 28 & \\
\hline Divorced, separated, widowed & $1140(31)$ & 31 & 16 & \\
\hline Single & $643(17)$ & 32 & 19 & \\
\hline Unknown & 138 (4) & 36 & 21 & \\
\hline Prior cancer & & & & $<0.001$ \\
\hline Yes & $553(15)$ & 28 & 14 & \\
\hline No & $3175(85)$ & 39 & 24 & \\
\hline Primary site & & & & $<0.001$ \\
\hline Tonsil & $1410(38)$ & 41 & 25 & \\
\hline Base of tongue & $1446(39)$ & 39 & 26 & \\
\hline Soft palate & $425(11)$ & 30 & 15 & \\
\hline Pharyngeal wall & $96 \quad(3)$ & 27 & 14 & \\
\hline Other & 351 & 29 & 14 & \\
\hline \multicolumn{5}{|l|}{ Grade } \\
\hline 1 & $233(6)$ & 36 & 18 & \\
\hline 2 & $1527(41)$ & 34 & 20 & \\
\hline 3 & $1274(34)$ & 43 & 28 & \\
\hline Unknown & 694 (19) & 37 & 19 & \\
\hline T stage & & & & $<0.001$ \\
\hline 1 & $471(13)$ & 47 & 26 & \\
\hline 2 & $1061(28)$ & 42 & 27 & \\
\hline 3 & $470(13)$ & 34 & 20 & \\
\hline 4 & 171 & 25 & 17 & \\
\hline Unknown & $1555(42)$ & 34 & 20 & \\
\hline Nodal stage & & & & $<0.001$ \\
\hline 0 & $1392(37)$ & 39 & 22 & \\
\hline 1 & 480 (13) & 41 & 25 & \\
\hline $2 \mathrm{a}$ & 218 & 43 & 31 & \\
\hline $2 b$ & 1007 (27) & 38 & 24 & \\
\hline $2 c$ & 495 (13) & 30 & 20 & \\
\hline 3 & 136 (4) & 24 & 15 & \\
\hline AJCC stage & & & & $<0.001$ \\
\hline I & $254 \quad(7)$ & 43 & 20 & \\
\hline II & 441 (12) & 43 & 29 & \\
\hline III & $606(16)$ & 42 & 25 & \\
\hline IVa & $1778(48)$ & 36 & 24 & \\
\hline $\mathrm{IVb}$ & 136 & 24 & 15 & \\
\hline Missing & $513(14)$ & 34 & 16 & \\
\hline
\end{tabular}


A

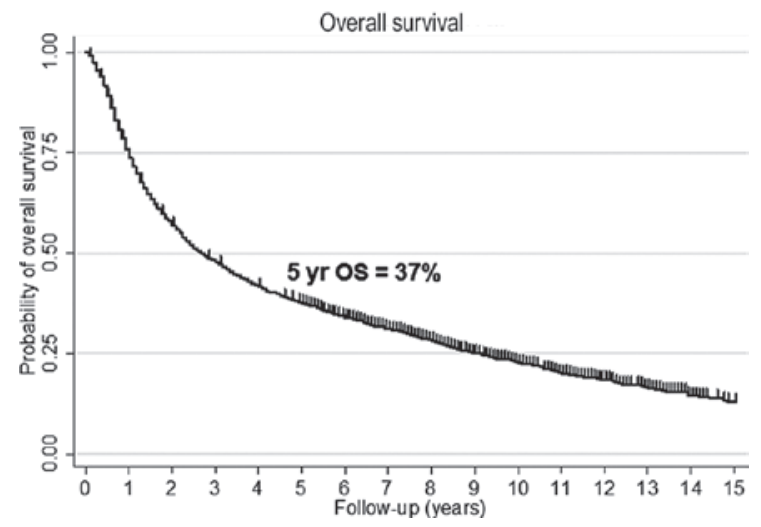

C

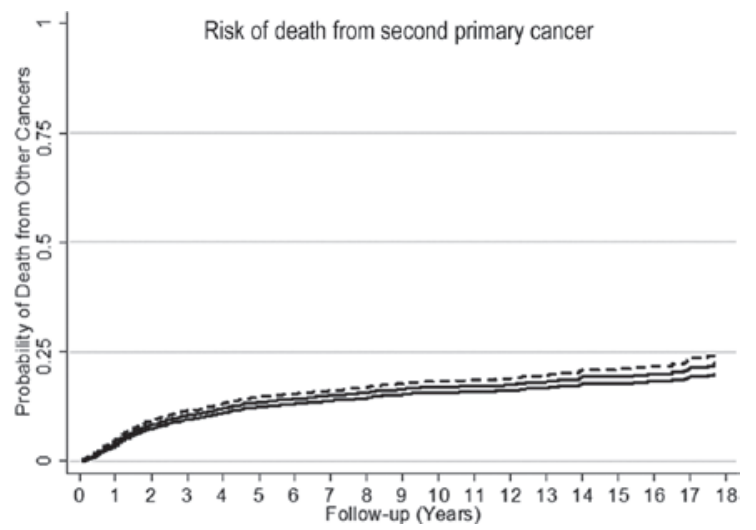

$\mathbf{E}$

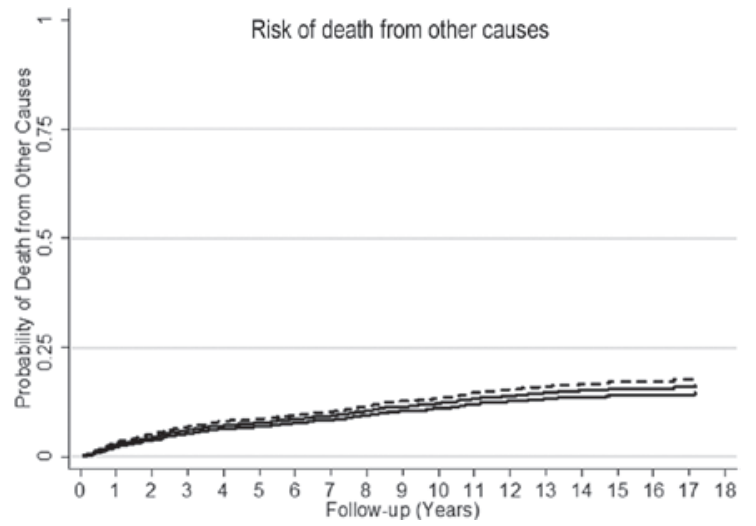

B

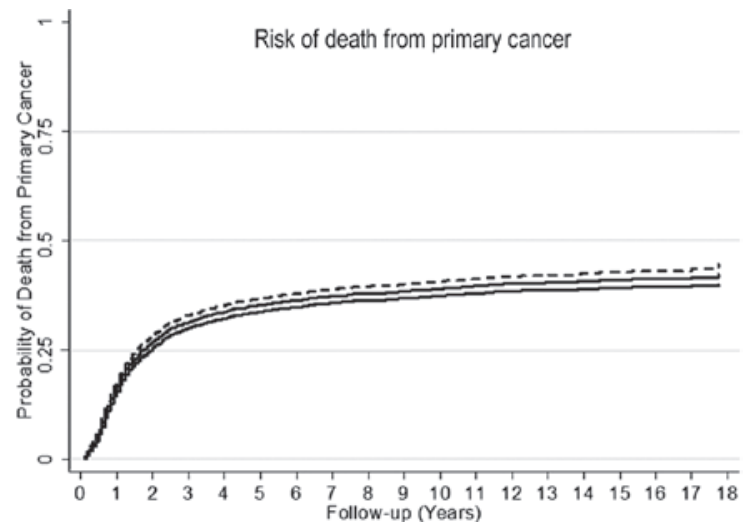

D

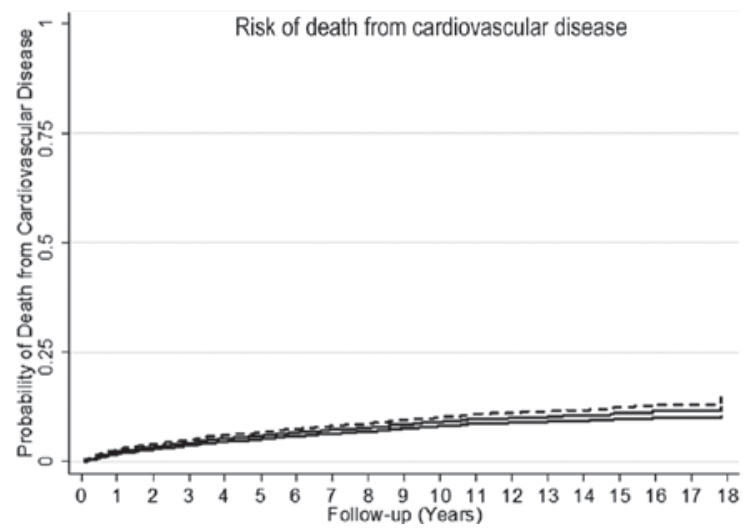

Figure 1. (A) Overall survival using the Kaplan-Meier method. (B) Risk of mortality from primary cancer using the cumulative incidence method. (C) Risk of mortality from second primary cancer using the cumulative incidence method. (D) Risk of mortality from cardiovascular disease using the cumulative incidence method. (E) Risk of mortality from other causes using the cumulative incidence method. OS, overall survival. Upper dotted lines and lower solid lines represent estimates of $95 \%$ confidence intervals.

over the past two decades, patients remain at significant risk of mortality from primary cancer $(1,5-6,8)$. Since the majority of deaths occurring in the first 5 years following diagnosis are attributed to primary cancer, further improvements in therapy would yield meaningful improvements in overall survival (19). The improved prognosis associated with oropharyngeal cancers in recent years may be related to increased utilization of concurrent chemoradiation, technological advances in radiation delivery and the increasing prevalence of HPV-related tumors $(5,9,12)$. Caucasian ethnicity and high grade tumors have both been associated with HPV-related tumors and likely account for some of the improved prognosis observed in those subgroups $(13,20)$. The 5 -year overall survival among patients of African descent was only $24 \%$. Consistent with previously published work, efforts to improve outcome in African-American patients by earlier diagnosis and improved treatment for HPV-negative disease are urgently required (20).

Treatment of elderly patients remains a challenge due to the increased difficulty of administering therapy to this cohort (21). The 5-year overall survival for the $>70$ year old population is $26 \%$. Two recent meta-analyses suggest that neither concurrent chemotherapy nor altered fractionation radiation therapy were beneficial for patients older than 70 years of age, at least partly due to the high risk of mortality from comorbid illness $(5,22)$. Whether better-tolerated combined modality treatments, such as concurrent cetuximab and radiotherapy, will improve outcome is currently unknown $(1,23)$. As expected, advanced $\mathrm{T}$ and $\mathrm{N}$ stage $(\geq \mathrm{N} 2 \mathrm{~B})$ predicted an increased risk of mortality from primary oropharyngeal cancer (24). Based on the historically poor survival of patients with T3-4 or N2c-N3 disease, good performance status patients with high-risk disease are an appropriate population of patients to investigate intensification of therapy beyond concurrent chemoradiotherapy by adding induction chemotherapy, altered fractionation radiation and/or biologically targeted therapy $(3,25-26)$. The association of 
Table II. Predictors of all cause mortality using Cox multivariable analysis.

\begin{tabular}{lcc}
\hline & HR & P-value \\
\hline Age (continuous) & 1.03 & $<0.001$ \\
African ethnicity & 1.44 & $<0.001$ \\
Female gender & 0.82 & 0.004 \\
Date of diagnosis (dichotomous) & 0.84 & 0.005 \\
Marital status & 0.75 & $<0.001$ \\
Subsite & 1.15 & 0.04 \\
First cancer diagnosis & 0.83 & 0.02 \\
T stage (continuous) & 1.16 & $<0.001$ \\
N stage (continuous) & 1.10 & $<0.001$ \\
Grade (continuous) & 0.85 & $<0.001$ \\
\hline
\end{tabular}

HR, hazard ratio.

unmarried status with mortality from primary oropharyngeal cancer is largely unexplained (16). Unmarried patients may lack social support and are at higher risk for pretreatment health behaviors such as tobacco and alcohol abuse, poor diet and limited physical activity (27).

Mortality from second primary cancer, cardiovascular disease and other causes. Between years 5 and 10 following diagnosis, $41 \%$ of 5 -year survivors succumbed to the disease. During this interval, $73 \%$ of mortalities were attributed to diagnoses other than oral cavity or oropharyngeal cancer. As the long-term survival of head and neck cancer continues to improve, identifying strategies to prevent late events may become increasingly important.

In contrast to field cancerization associated with tobaccoand alcohol-related upper aerodigestive tumors, oral HPV infection is generally focal (13). Emerging data suggest that
HPV-related patients have a lower risk of second malignancies (14). Consistent with this theory, patients more likely to harbor HPV-positive tumors, such as those of younger age, female gender, tonsil or base of tongue subsite and high-grade disease, have a lower risk of mortality from second cancer (13). Given the increased risk of cancer associated with tobacco use, prior diagnosis of cancer may be a surrogate for smoking status (28). In this study, we also identified a cohort of patients at increased risk of cardiovascular disease and mortality from other causes. Several risk factors overlap with those associated with second cancers (male gender and prior cancer diagnosis), indicating that tobacco use is the common risk factor. African-American patients and the elderly appear to be at particularly high risk for mortality from cardiovascular disease. Increased surveillance and optimal medical management appear appropriate for these subgroups (29).

Limitations. It is important to acknowledge the limitations of this retrospective analysis of the SEER registry. Several important factors, including HPV status, performance status, concurrent administration of chemotherapy and details of radiation delivery, are not available for analysis. Accurate attribution of cause of mortality is always a potential source of error. Within the limitations of diagnosis codes, it is not always possible to accurately classify mortality from primary or secondary cancer. For this analysis, we classified mortality from oral cavity or oropharyngeal cancer as primary disease. This is necessary since in the SEER site recode schema, base of tongue cancer is subclassified under oral cavity rather than oropharyngeal cancer. Despite these limitations, these data provide useful insight into the competing risks of mortality of patients with oropharyngeal cancer and may help to assist with future clinical trial design for this patient population.

In conclusion, although the prognosis of patients with oropharyngeal cancer treated with radiotherapy has improved in recent years, the 5-year overall survival remains below $50 \%$. Earlier diagnosis and more effective cancer therapy is required to further improve the 5-year survival, while

Table III. Predictors of cause-specific mortality using Cox multivariable analysis.

\begin{tabular}{|c|c|c|c|c|c|c|c|c|}
\hline & $\begin{array}{c}\text { Primary cancer } \\
\text { HR }\end{array}$ & P-value & $\begin{array}{c}\text { Second cancer } \\
\text { HR }\end{array}$ & P-value & $\begin{array}{c}\text { CV disease } \\
\text { HR }\end{array}$ & P-value & $\begin{array}{c}\text { Other causes } \\
\text { HR }\end{array}$ & P-value \\
\hline Age (continuous) & 1.03 & $<0.001$ & 1.02 & $<0.001$ & 1.09 & $<0.001$ & 1.04 & $<0.001$ \\
\hline African ethnicity & 1.48 & $<0.001$ & 1.12 & 0.55 & 2.02 & 0.004 & 1.39 & 0.14 \\
\hline Female gender & 1.02 & 0.78 & 0.63 & 0.002 & 0.56 & 0.004 & 0.75 & 0.08 \\
\hline $\begin{array}{l}\text { Date of diagnosis } \\
\text { (dichotomous) }\end{array}$ & 0.82 & 0.02 & 0.88 & 0.37 & 0.83 & 0.27 & 0.87 & 0.36 \\
\hline Marital status & 0.75 & $<0.001$ & 0.83 & 0.16 & 0.89 & 0.50 & 0.60 & 0.001 \\
\hline Subsite & 0.93 & 0.47 & 1.85 & $<0.001$ & 1.14 & 0.51 & 1.19 & 0.30 \\
\hline First cancer diagnosis & 1.15 & 0.26 & 0.51 & $<0.001$ & 0.60 & 0.01 & 0.84 & 0.37 \\
\hline T stage (continuous) & 1.28 & $<0.001$ & 1.10 & 0.17 & 0.93 & 0.49 & 1.03 & 0.75 \\
\hline $\mathrm{N}$ stage (continuous) & 1.15 & $<0.001$ & 1.07 & 0.10 & 1.03 & 0.62 & 1.02 & 0.70 \\
\hline Grade (continuous) & 0.79 & $<0.001$ & 0.81 & 0.03 & 1.11 & 0.42 & 0.96 & 0.73 \\
\hline
\end{tabular}

HR, hazard ratio; CV disease, cardiovascular disease. 
reducing mortality from other causes may impact the 10 -year survival.

\section{References}

1. Bonner JA, Harari PM, Giralt J, et al: Radiotherapy plus cetuximab for squamous-cell carcinoma of the head and neck. $N$ Engl J Med 354: 567-578, 2006.

2. Fu KK, Pajak TF, Trotti A, et al: A Radiation Therapy Oncology Group (RTOG) phase III randomized study to compare hyperfractionation and two variants of accelerated fractionation to standard fractionation radiotherapy for head and neck squamous cell carcinomas: first report of RTOG 9003. Int J Radiat Oncol Biol Phys 48: 7-16, 2000.

3. Posner MR, Hershock DM, Blajman CR, et al: Cisplatin and fluorouracil alone or with docetaxel in head and neck cancer. $\mathrm{N}$ Engl J Med 357: 1705-1715, 2007.

4. Shiboski CH, Schmidt BL and Jordan RC: Tongue and tonsil carcinoma: increasing trends in the U.S. population ages 20-44 years. Cancer 103: 1843-1849, 2005.

5. Bourhis J, Overgaard J, Audry H, et al: Hyperfractionated or accelerated radiotherapy in head and neck cancer: a metaanalysis. Lancet 368: 843-854, 2006.

6. Denis F, Garaud P, Bardet E, et al: Final results of the 94-01 French Head and Neck Oncology and Radiotherapy Group randomized trial comparing radiotherapy alone with concomitan radiochemotherapy in advanced-stage oropharynx carcinoma. J Clin Oncol 22: 69-76, 2004.

7. Garden AS, Harris J, Vokes EE, et al: Preliminary results of Radiation Therapy Oncology Group 97-03: a randomized phase II trial of concurrent radiation and chemotherapy for advanced squamous cell carcinomas of the head and neck. J Clin Oncol 22: 2856-2864, 2004.

8. Kao J, Lavaf A, Teng MS, Huang D and Genden EM: Adjuvant radiotherapy and survival for patients with node-positive head and neck cancer: an analysis by primary site and nodal stage. Int J Radiat Oncol Biol Phys 71: 362-370, 2008.

9. Lee NY, de Arruda FF, Puri DR, et al: A comparison of intensitymodulated radiation therapy and concomitant boost radiotherapy in the setting of concurrent chemotherapy for locally advanced oropharyngeal carcinoma. Int J Radiat Oncol Biol Phys 66 966-974, 2006.

10. Mell LK, Dignam JJ, Salama JK, et al: Predictors of competing mortality in advanced head and neck cancer. J Clin Oncol 28 15-20, 2010.

11. Argiris A, Brockstein BE, Haraf DJ, et al: Competing causes of death and second primary tumors in patients with locoregionally advanced head and neck cancer treated with chemoradiotherapy. Clin Cancer Res 10: 1956-1962, 2004

12. Vu HL, Sikora AG, Fu S and Kao J: HPV-induced oropharyngeal cancer, immune response and response to therapy. Cancer Lett 288: 149-155, 2009.

13. Gillison ML: Human papillomavirus and prognosis of oropharyngeal squamous cell carcinoma: implications for clinical research in head and neck cancers. J Clin Oncol 24: 5623-5625, 2006.

14. Licitra L, Perrone F, Bossi P, et al: High-risk human papillomavirus affects prognosis in patients with surgically treated oropharyngeal squamous cell carcinoma. J Clin Oncol 24: 5630-5636, 2006.
15. D'Souza G, Kreimer AR, Viscidi R, et al: Case-control study of human papillomavirus and oropharyngeal cancer. $\mathrm{N}$ Engl J Med 356: 1944-1956, 2007.

16. Lavaf A, Genden EM, Cesaretti JA, Packer S and Kao J: Adjuvant radiotherapy improves overall survival for patients with lymph node-positive head and neck squamous cell carcinoma. Cancer 112: 535-543, 2008.

17. Coviello V and Boffess M: Cumulative incidence estimation in the presence of competing risks. Stata J 4: 103-112, 2004.

18. Kim HT: Cumulative incidence in competing risks data and competing risks regression analysis. Clin Cancer Res 13: 559-565, 2007.

19. Haddad RI and Shin DM: Recent advances in head and neck cancer. N Engl J Med 359: 1143-1154, 2008.

20. Settle K, Posner MR, Schumaker LM, et al: Racial survival disparity in head and neck cancer results from low prevalence of human papillomavirus infection in black oropharyngeal cancer patients. Cancer Prev Res (Phila Pa) 2: 776-781, 2009.

21. Genden EM, Rinaldo A, Shaha AR, et al: Treatment considerations for head and neck cancer in the elderly. J Laryngol Otol 119: 169-174, 2005.

22. Pignon JP, le Maitre A, Maillard E and Bourhis J: Meta-analysis of chemotherapy in head and neck cancer (MACH-NC): an update on 93 randomised trials and 17,346 patients. Radiother Oncol 92: 4-14, 2009.

23. Kao J, Sikora AT and Fu S: Dual EGFR and COX-2 inhibition as a novel approach to targeting head and neck squamous cell carcinoma. Curr Cancer Drug Targets 9: 931-937, 2009.

24. Greene FL, American Joint Committee on Cancer. and American Cancer Society: AJCC cancer staging manual. Springer, New York, 2002

25. Pfister DG, Su YB, Kraus DH, et al: Concurrent cetuximab, cisplatin, and concomitant boost radiotherapy for locoregionally advanced, squamous cell head and neck cancer: a pilot phase II study of a new combined-modality paradigm. J Clin Oncol 24: 1072-1078, 2006.

26. Salama JK, Stenson KM, Kistner EO, et al: Induction chemotherapy and concurrent chemoradiotherapy for locoregionally advanced head and neck cancer: a multi-institutional phase II trial investigating three radiotherapy dose levels. Ann Oncol 19: 1787-1794, 2008.

27. Duffy SA, Ronis DL, McLean S, et al: Pretreatment health behaviors predict survival among patients with head and neck squamous cell carcinoma. J Clin Oncol 27: 1969-1975, 2009.

28. Khuri FR, Lee JJ, Lippman SM, et al: Randomized phase III trial of low-dose isotretinoin for prevention of second primary tumors in stage I and II head and neck cancer patients. J Natl Cancer Inst 98: 441-450, 2006.

29. Pearson TA, Blair SN, Daniels SR, et al: AHA guidelines for primary prevention of cardiovascular disease and stroke: 2002 update: consensus panel guide to comprehensive risk reduction for adult patients without coronary or other atherosclerotic vascular diseases. American Heart Association Science Advisory and Coordinating Committee. Circulation 106: 388-391, 2002. 